motivation; work, work community and leadership. Evidence shows that health, functional capacities and work (community) affect work-ability the most. Work-ability and both latter factors were therefore assessed in a large sample of employees and compared between younger and older workers.

Methods A cross-sectional study was performed in 100 Belgian companies. The online questionnaire 'Wellfie' was used, which is based on the 'house of work-ability'. The tool consists of validated scales assessing work-ability using four questions of the work-ability index (Range: 1: very bad to 5: very good), lifestyle (diseases) and physical burden. Descriptive statistics were performed using frequencies and equality of proportions was analysed using Chi-square.

Result 3887 participants completed Wellfie (i.e. 67\% female, $33 \%$ male; $60 \%$ age $<45,40 \%$ age $\geq 45$ ). Their current work-ability was similar (Mean: 3.90), yet the predicted workability in the upcoming two years was significantly lower in the upper age group (Mean: 4.10 versus 3.95; $\mathrm{p}<0.05$ ). Employees of 45 years or more reported more musculoskeletal disorders affecting their work (27.7\% versus $16.7 \%)$, episodes of burnout or depression $(17.5 \%$ versus $13 \%$; $<<0.05)$ and lifestyle diseases such as diabetes $(2.4 \%$ versus $1.1 ; \mathrm{p}<0.05)$, arterial hypertension $(25.9 \%$ versus $10 \% ; \mathrm{p}<0.05)$ and cardiovascular diseases $(10 \%$ versus $4.6 ; \mathrm{p}<0.05)$. The reported amount of physical burden (e.g. repetitive movements, lifting) is comparable between age groups.

Discussion Ageing goes along with a higher risk for chronic diseases and comorbidities affecting employee's work-ability. Employers should therefore invest more in a health promotion policy for all workers.

\section{DETERMINANTS OF EARLY RETIREMENT AMONG OLDER WORKERS WITH AND WITHOUT CHRONIC DISEASE: RESULTS FROM A DANISH PROSPECTIVE STUDY}

${ }^{1}$ Ranu Sewdas, ${ }^{2}$ Sannie Vester Thorsen, ${ }^{1}$ Cécile RL Boot, ${ }^{2}$ Jakob Bue Bjørner, ${ }^{1}$ Allard J van der Beek. 'Amsterdam Public Health research institute, VU University Medical Centre, Amsterdam, The Netherlands; ${ }^{2}$ The Danish National Research Centre for the Working Environment, Copenhagen, Denmark

\subsection{6/oemed-2018-ICOHabstracts. 130}

Introduction Previous studies have found that several factors are associated with early retirement among older workers. Given the high prevalence of chronic diseases among older workers the aims of this study were to explore:

- the determinants of early retirement among older workers with and without chronic diseases, and

- whether these determinants differed between workers with and without chronic diseases.

Methods Danish workers aged 56-64 years, who were member of the early retirement scheme were selected from the Danish National Working Environment Survey, and were followed in a public register for four years. Cox-regression analyses were performed separately for those with and without chronic diseases to determine the associations between the determinants (health, work-related, and social factors) and early retirement. To explore differences, an interaction term between the determinant and having a chronic disease was included among the analyses on the total population.

Result Among workers with chronic diseases, poor health (HR 2.15; 95\% CI: 1.37 to 3.37), more depressive symptoms
(1.01; 1.00-1.03), high physical workload (1.84; 1.37-2.48), low job satisfaction $(3.08 ; 2.09-4.55)$, low influence at work (1.94; 1.36-2.77), and work-family conflict $(1.01 ; 1.00-1.01)$ were associated with early retirement. Among those without chronic diseases, poor health $(2.56 ; 1.27-5.16)$, more depressive symptoms (1.03; 1.01-1.05), high physical workload (2.09; 1.39-3.13), low job satisfaction (5.27; 2.96-9.40), low influence at work $(1.69 ; 1.04-2.75)$, and poor relationship with colleagues $(2.81 ; 1.44-5.49)$ were associated with early retirement. None of the interactions were found to be statistically significant $(\mathrm{p}>0.05)$.

Discussion Prolonged work participation of older workers is a challenge for governments in high-income countries. The findings from this study indicate that determinants that might influence prolonged work participation among older workers are not distinct for those with and without chronic diseases. Interventions aiming at work participation until an older age might be the same for both groups.

\section{HEALTHY EATING CHOICES: EMPLOYEE PERCEPTIONS OF THE ROLE OF THE EMPLOYER}

${ }^{1} J$ udith Grant*, 'Jonathan Houdmont, ${ }^{2}$ Fehmidah Munir, ${ }^{3}$ Robert Kerr. ${ }^{1}$ University of Nottingham, Nottingham, UK; ${ }^{2}$ Loughborough University, Loughborough, UK; ${ }^{3}$ Ulster University, Newtownabbey, UK

\subsection{6/oemed-2018-ICOHabstracts. 131}

Introduction Changes in lifestyles and diet over the last 30 years have led to a significant rise in overweight and obesity. By the year 2020 seven out of ten people in Britain may be overweight or obese, putting a strain on health services and increasing costs to the economy. Organisations may contribute to poor health behaviours, however they are also well placed to support employees; therefore understanding employees' perceptions of the barriers and facilitators to healthy eating at work may assist in designing interventions to address these.

Methods This qualitative study was conducted in 2017 using semi-structured telephone interviews in a large organisation that had recently been privatised after many decades in public ownership. Demographic information collected from participants included three indices of SES (education, income and job type), age, gender, dependents, and perceived weight status. Participants were asked to consider the barriers and facilitators to eating a healthy well-balanced diet and their perceptions of the role of the employer in promoting healthy eating. Thematic analysis was carried out using Braun and Clarke's (2006) methodology and analysis carried out through the computer software NVivo.

Result Thematic saturation was reached upon completion of 15 interviews. Five main themes were identified, each containing multiple sub-themes:

- Knowledge (fruit and vegetable portions; source of knowledge),

- Behaviour (cost of food influencing purchasing; eating past the point of feeling full; motivation to eat a healthily),

- Access (access to healthy foods in the workplace; preparing food in advance),

- Workplace Culture (long hours and travel; taking breaks; workplace temptation; leadership behaviours) and 5 .

Responsibility (Government and organisational responsibilities). 
Discussion Workplace culture was seen as a barrier to healthy eating. Initiatives designed to modify work culture may prove effective as a means by which to promote healthy eating in the organisational setting.

\section{THE ROLE OF AGE AND HEALTH IN RETURNING TO WORK: RESULTS FROM THE SUPPORTING OLDER PEOPLE INTO EMPLOYMENT (SOPIE) COHORT}

1J Brown*, ${ }^{2}$ SV Katikireddi, ${ }^{2}$ AH Leyland, ${ }^{3}$ RW McQuaid, ${ }^{4} \mathrm{~J}$ Frank, ${ }^{1}$ EB Macdonald. ${ }^{1}$ Healthy Working Lives Group, Institute of Health and Wellbeing, University of Glasgow; ${ }^{2} \mathrm{MRC} / \mathrm{CSO}$ Social and Public Health Sciences Unit, Institute of Health and Wellbeing, University of Glasgow; ${ }^{3}$ Stirling Management School, University of Stirling, Stirling; ${ }^{4}$ Scottish Collaboration for Public Health Research and Policy, University of Edinburgh

\subsection{6/oemed-2018-ICOHabstracts. 132}

Introduction By 2020 people aged 50 years and over will make up almost half of the adult population in the UK. Policy aims to enable more people to work for longer however there is a dramatic drop in labour participation after age 50. Our aim was to investigate the impact of age, and health on return to work (RTW) in welfare benefit claimants engaging with the Work Programme (WP); the UK Government's main RTW initiative. It supports two main groups of claimants for two years - Job Seeker Allowance (JSA), for people who are unemployed but capable of work; Employment Support Allowance (ESA), for people with a disability that makes it more difficult to work.

Methods The data were from the SOPIE cohort (13 461 unemployed clients aged 18-64, who entered the WP in Scotland in 2013/2014). Data were analysed using STATA 14 and a Poisson modelling approach using fractional polynomials to model age as a continuous variable.

Results Clients aged 50 and over accounted for 15\% of JSA and $30 \%$ ESA groups. The proportion of clients disclosing health conditions (HC) were: 'JSA under-50', 25\%; 'JSA over50', 53\%; 'ESA under-50', 97\%; 'ESA over-50', 98\%. Multiple $\mathrm{HC}$ were more common in ESA clients. Job start rates for clients were: 'JSA under-50', 65\%; 'JSA over-50', 49\%; 'ESA under-50', 23\%; 'ESA over-50', 14\%. There was a strong relationship between age, health and job start with the predicted probability of job start highest in the first three months of the WP. The analyses also investigated the influence of biopsychosocial factors on RTW.

Conclusion This study is on-going and will inform interventions focussing on addressing age-specific, health and biopsychosocial barriers for future RTW programmes with the aim of improving employment outcomes, so that not only individuals but employers and the economy can benefit from extending working lives.

\section{PHYSICAL REHABILITATION APPROACH FOR RETAINING HEALTH CARE WORKERS SUFFERING FROM MUSCULOSKELETAL DISORDERS} ${ }^{1}$ School of Specialisation in Occupational Health, University of Milan, Milan, Italy; ${ }^{2}$ Occupational Health Unit, San Gerardo Hospital, Monza, Italy; ${ }^{3}$ School of Medicine and Surgery, University of Milan Bicocca, Milan, Italy

\subsection{6/oemed-2018-ICOHabstracts. 133}

Introduction Healthcare professionals are known to be at high risk for work-related musculoskeletal disorders (MSDs). Physical rehabilitation may be an important approach for retaining
Health Care Workers (HCWs) suffering from these disorders, especially among older workers. The aim of this study was to evaluate the results of a rehabilitation program dedicated to the employees of a large hospital in the Northern Italy.

Methods HCWs with shoulder disorders (SD) and Low Back Pain (LBP) were identified by the Occupational Health Unit and admitted to a physical rehabilitation program. Functional assessment scales were administered at the beginning and the end of the program: UCLA and Constant scale for SD and Borg scale for LBP. We applied Wilcoxon test for statistical comparisons. The level of significance adopted was 5\%.

Results During a 24 months period, occupational physicians identified $123 \mathrm{HCWs}$ with MSDs. Workers were mainly nurses (37.5\%) and nursing assistive personnel (34.8\%), with an average length of service of $28.53 \pm 8.92$ years. Only 47 workers completed the rehabilitation program. The treated workers were mainly affected by LBP $(n=22,46.81 \%)$ and SD $(n=20,42.52 \%)$. After rehabilitation, significant improvements in the UCLA scale ( $p$-value: 0.0206) were observed among the subjects affected by SD. The improvements in the Borg scale were also significant (p-value: 0.0011) among the subjects affected by LBP. Occupational physicians prescribed work restrictions in only five subjects $(5.88 \%)$. Three workers with previous work restrictions were considered fully fit-forwork after treatment. The remaining subjects returned to work without any restrictions.

Conclusions A rehabilitation program appears to be a valuable approach for retaining older HCWs affected by MSDs. In our study, more than $88 \%$ of workers have positively evaluated the program. Occupational physicians may play an important role in this program, especially in the assessment of subjects with work-related problems.

\section{IMPACT OF INDIVIDUAL MOTIVATION DIFFERENCES ON REDUCING SEDENTARY BEHAVIOUR}

1,2 V Hermans* ${ }^{*}{ }^{2} \mathrm{~L}$ Van Naemen, ${ }^{3} \mathrm{~J}$ Seghers, $1,{ }^{4} \mathrm{~L}$ Godderis, 1,5 L Daenen. ${ }^{1} K$ nowledge, Information and Research Center, IDEWE Group (External Service for Prevention and Protection at Work), Leuven, Belgium; '2Department of Experimental and Applied Psychology, Work and Organisational Psychology (WOPS), Faculty of Psychology and Education Sciences Vrije Universiteit Brussel, Brussel, Belgium; ${ }^{3}$ Department of Kinesiology, Physical Activity, Sports \& Health Research Group, KU Leuven, Leuven, Belgium; ${ }^{4}$ Environment and Health, Faculty of Medicine, KU Leuven, Louvain, Belgium; ${ }^{5}$ Department of Rehabilitation Sciences and Physiotherapy, Human Physiology and Anatomy (KIMA), Faculty of Physical Education and Physiotherapy, Vrije Universiteit Brussel, Brussels, Belgium

\subsection{6/oemed-2018-ICOHabstracts.134}

Introduction Reviews small effects for interventions sitting behaviour. This study aimed at evaluating basic psychological needs (need for competence, for autonomy and for relatedness) reduction in sitting behaviour.

Methods This study is part of the Move@TheOffice RCTstudy in the offices a large pharmaceutical company. The experimental group (19 participants) received a multiintervention to reduce sitting. To measure the basic psychological needs, the Work-related Basic eed atisfaction cale was used. The BREQ-3 measured the degree of motivation regulation to reduce sitting. Sitting was measured using the micro Activ$\mathrm{Pal}^{\mathrm{TM}}$ monitor. Data were analysed using SPSS.

Results Significant decrease $(p<0,05)$ in sitting time was found the experimental group. A significant BREQ-index was found after the intervention $(p<0.05)$, a higher to decrease sitting 